\title{
Psychological aspects of temporomandibular disorders - literature review
}

\author{
Marcin Berger ${ }^{1}$, Justyna Oleszek-Listopad², Milena MarczaK³, Jolanta Szymanska ${ }^{4 *}$ \\ ${ }^{1}$ Department of Functional Masticatory Disorders, Medical University of Lublin, Poland \\ ${ }^{2}$ Department of Dental Prosthetics, Medical University of Lublin, Poland \\ ${ }^{3}$ General Psychology Department, Institute of Psychology, Maria Curie-Sklodowska University, Lublin, Poland \\ ${ }^{4}$ Chair and Department of Paedodontics, Medical University of Lublin, Karmelicka 7, 20-081 Lublin, Poland
}

\section{ARTICLE INFO \\ Received 02 March 2015 \\ Accepted 26 March 2015}

\section{Keywords:}

temporomandibular disorders,

psychological stress,

depression,

anxiety.

\begin{abstract}
Temporomandibular disorders (TMD) constitute a group of clinical problems involving the masticatory muscles, the temporomandibular joint and associated structures. An etiological connection of TMD with psychological factors was proposed as early as the 1980's. Indeed, the interdependence of psychological and health aspects in the patient's treatment, place light upon the more important variables contributing to the various mental disorders that may accompany TMD. Current literature suggests a close relationship between TMD and selected psychological factors, such as personality traits, stress, depression, anxiety, and catastrophizing. Of note, anxiety-depressive disorders, somatisation and catastrophizing contribute to chronic TMD, mainly in the form of myofascial pain. Hence, knowledge of the influence of psychological factors affecting TMD, enables the identification of patients with an increased risk of chronic painful TMD.
\end{abstract}

\section{INTRODUCTION}

The term temporomandibular disorders (TMD) embraces a grouping of clinical problems involving the masticatory muscles, the temporomandibular joint and associated structures [4]. The more frequently reported symptoms related to TMD include pain, acoustic symptoms in the temporomandibular joints (TMJ), and disorders in mandibular movements. TMD form a group of disorders of highly heterogenous etiology. Traumas, occlusal disorders and parafunctional habits are most frequently reported as factors that may cause TMD. An etiological connection of TMD with psychological factors was proposed as early as 1980's. Discussions on the interdependence of psychological and health aspects in the patient's treatment, aim at presenting the variables of greater importance that contribute to the varied mental disorders that may accompany TMD. The relationship between psychological factors and TMD is multidirectional. The current state of mentality may be an effect, but also a cause of the emergence, continuation or exacerbation of TMD, which makes correct assessment difficult. This demonstrates the need to understand the physical

\footnotetext{
* Corresponding author

e-mail: szymanska.lublin@gmail.com
}

and psychological characteristics of an individual patient. Such differences depend on heredity, sex, and social-natural environment. They influence the functioning of an individual and are manifested in his or her personality [21]. Hence, the degree of contribution of different factors to TMD may be related to individual differences among people.

The interaction between TMD and mental state is particularly clear in patients suffering from the complaints involving the limitation of the basic functions of the masticatory motor system, both biological (chewing) and interpersonal (speech, emotional expression). One of the predominant symptoms, and at the same time the main cause of the patients reporting for treatment due to TMD, is a pain described by the International Association for the Study of Pain as an unpleasant sensory and emotional experience associated with actual or potential tissue damage, or described in terms of such damage [29]. According to the cited definition, pain is a mental, emotional and subjective phenomenon. To obtain a complete picture of a pain disorder, it is necessary to apply in its examination the socalled biopsychosocial model of pain [23], which apart from biological factors, includes also psychological and social aspects/variables related to the disease. The biopsychosocial model was introduced to TMD diagnostics on a large scale 
through the Research Diagnostic Criteria (RDC) published in 1992 [6]. The RDC questionnaire, in addition to clinical examination (axis I), includes psychometric tools that enable the assessment of the patient's psychological parameters (axis II), as well as the questions necessary for the evaluation of his or her social situation. Currently, this model is used in the examination of all craniofacial pain disorders [36].

\section{AIM}

By way of a review of current literature, the paper advances the relationship between TMD and selected psychological factors, such as personality traits, stress, depression, anxiety, and catastrophizing.

\section{The connection of psychological factors to functional disorders}

Numerous studies conducted over the recent twenty years have noted a connection between several psychological variables and TMD $[8,10,11]$. Indeed, the differences in the intensity of personality traits, experienced stress level, depression and catastrophizing between TMD patients and individuals without this disorder can be readily observed. A noted example of the interconnection of psychosocial risk factors in chronic TMD is the presence of a lacking of a sense of security that accompanies a patient's prolonged experience of suffering [11]. Beyond this, it is also essential to realize that there are psychological factors associated with initial pain symptoms in TMD, as well as sociodemographic variables that contribute to the experience of craniofacial pain $[8,10,11]$. One of the more important determinants influencing the behaviour and functioning of an individual is his or her personality vis a vis his or her holding of five personal traits: neuroticism, extraversion, openness to experience, agreeableness, and conscientiousness [28]. It is also important to note that the intensity of the expression of the aforementioned traits is crucial in their expression of individual personality.

\section{Personality type}

Personality is the dynamic organization within the individual of the psychophysical systems that are decisive for his or her functioning within that individual's current environment and which determine his or her behaviour. In an adult individual, such traits are relatively constant.

Some personality traits are expressed by way of bruxism, defined as the repeated jaw muscle activity manifested as the clenching of the jaw, the grinding and/or stiffening or protrusion of the mandible. There are two types of bruxism: sleep bruxism and awake bruxism [22]. Bruxism is related to the disorders of both the temporomandibular joint and the masticatory muscles [25]. Pingitore et al. found that it occurred more frequently in ambitious, aggressive, rivalry-prone and emotionally highly-strung individuals [32]. However, Fisher and O'toole showed that bruxers are characterized by a withdrawn, avoiding and shy attitude [12].

\subsection{Neuroticism}

Neuroticism is a personality dimension that indicates the emotional adaptation level of an individual. People with a large intensity of this trait are prone to great anxiety and tension. They also find it difficult to cope with stressful situations and experience an anger and anxiety that is combined with low self-esteem [28]. High neuroticism is often associated with pathological symptoms and general malaise. Neurotic persons show sensitization tendencies, such as exaggerating physical discomfort and psychological problems, while individuals with a low neuroticism level show a tendency to deny health problems [5].

According to Moayedi et al., neuroticism may contribute to TMD pathophysiology, because there is a correlation between chronic pain in TMD and the patient's neurotic personality [30]. Neuroticism is related to muscular TMD [31].

\section{Stress}

Stress is a state that results from an imbalance between demands and resources, accompanied by strong negative emotions. The affect may be associated with physiological and biochemical responses that exceed the resting-state arousal level. Stress may be both dangerous and mobilizing for a person. Of note, if it is chronic and prolonged, it disturbs the normal function of the system. Stress affects not only the psyche, but also is made evident through certain symptoms expressed in various systems of the organism, including the masticatory motor system (Table 1).

Table 1. Stress response according to Selye [37]

\begin{tabular}{|l|l|}
\hline $\begin{array}{l}\text { Stage I } \\
\text { Alarm stage }\end{array}$ & $\begin{array}{l}\text { In response to a stressor, the organism is mobilized to } \\
\text { cope with the problem. At this stage, the organism's } \\
\text { immune system is weakened. }\end{array}$ \\
\hline $\begin{array}{l}\text { Stage II } \\
\text { Resistance stage }\end{array}$ & $\begin{array}{l}\text { The shock subsides. The organism adapts to } \\
\text { a persisting stressor by changing organ functions. }\end{array}$ \\
\hline $\begin{array}{l}\text { Stage III } \\
\text { Exhaustion stage }\end{array}$ & $\begin{array}{l}\text { This comes about when a stressor is too strong or } \\
\text { the exposure to it lasts too long, and the organism } \\
\text { cannot cope with it. This stage is associated with all } \\
\text { the negative health aspects being expressed. }\end{array}$ \\
\hline
\end{tabular}

Emotions characteristic for particular stress states are related to the occurrence of certain physiological changes, which, in turn, may facilitate or accelerate the development of a somatic disease. A prolonged state of emotional tension often induces an increased muscle activity, including that of the masticatory muscles. What is more, chronic tension is associated with an increase in parafunctional activity, which is considered to be one of the main TMD factors [38]. Parafunctions are defined as unconscious, abnormal and habitual activities of the masticatory organ outside its normal function. They may occur both during wakefulness and during sleep. Stress, as well as anxiety, depression and personality traits exert an important influence on the increased frequency of parafunctional teeth contact [13]. Numerous studies confirm the association of stress with bruxism during wakefulness, but there are no reliable data to confirm the connection of stress with bruxism during sleep [26].

\subsection{Styles of coping with stress}

Coping styles are defined as cognitive and behavioural efforts to recover homeostasis between demands and the resources of an individual in a situation he or she perceives as being burdensome and exceeding with regard to their current available resources. The adoption of a particular coping styles is an attempt to eliminate or reduce the 
experienced state of stress. The assumed style of coping with stress is related to the individual differences between people, and depends on personality traits, demographic characteristics (age, sex, education), current psychophysical status and the particular stress situation itself [27].

Table 2. Possible coping styles in particular stress situations [37]

\begin{tabular}{|l|l|}
\hline Task-oriented & $\begin{array}{l}\text { In stress-filled situations, an individual undertakes } \\
\text { certain tasks to surmount the problem, by way of } \\
\text { attempting to modify or change the situation. This } \\
\text { coping style is expressed by way of planning and } \\
\text { problem solving. }\end{array}$ \\
\hline Emotion-oriented & $\begin{array}{l}\text { In stress-filled situations, an individual self-focuses } \\
\text { emotionally, and recurs to wishful thinking so as to } \\
\text { reduce the tension related to a given stress situation. }\end{array}$ \\
\hline Avoidance-oriented & $\begin{array}{l}\text { In stress-filled situations, an individual represses } \\
\text { the thoughts concerning the current state of being } \\
\text { by performing substitute activities, e.g. watching } \\
\text { television, sleeping or practicing particular social } \\
\text { contacts. }\end{array}$ \\
\hline
\end{tabular}

Reissmann et al. found that the choice of stress-related adaptive coping style is associated with the pain diagnosed in TMD. They also concluded that knowledge of the coping style adopted by the patient is crucial for inducing appropriate clinical decisions and selecting alternative methods of disease treatment [33]. Other factors involved in abnormal neurotransmission of pain, contributing to pain fixation, include depression, anxiety and catastrophizing [24]. In patients with chronic painful TMD, the intensity, duration and localization of pain depend on the concurrent anxiety, depression and somatization [16].

\section{Depression}

Depression is classified as a mental health factor. The most frequently evidenced symptoms of depression include: negative mood, loss of interests, decreased activity or resistance to fatigue, concentration difficulties, low self-esteem, sleep problems and pessimism [39].

The concurrence of depression and numerous pain disorders, including painful TMD, has been well documented [7, $19,20]$. In patients with depression and a concurrent pain disorder, pain intensity and function limitation is greater than in people who do not suffer from depression. In addition, pain treatment is usually less effective in depressive patients. The interaction of pain and depression is probably mutual, and there are numerous theories put forward to explain their concurrence. For instance, this interaction may be explained by way of neurobiological factors, as the same neurotransmitters are responsible for pain transmission and mood maintenance [15]. Moreover, patients with TMD suffering from stress and depression show an increased activity of the masticatory muscles, as well as an increase in parafunctional teeth contacts $[3,13]$.

The study by Lajnert et al. on patients with chronic TMD reveals that the disease concurs with the symptoms of depression and somatisation. This may also lower pain and stress tolerance level. A higher level of depression and somatisation was also found in patients with painful TMD, in comparison to patients with acute TMD symptoms [20]. Similar studies demonstrate that there is a close connection between pain experience in TMD and a high depression level, as well as tinnitus [9].
According to Kindler et al., diagnosis, prevention and treatment of TMD pain must take into consideration depression and anxiety symptoms [19].

\section{Anxiety}

Research shows that anxiety disorders are intrinsically related to painful TMD [34]. In ICD-10 classification, anxiety can be categorized as being a neurotic disorder [39].

\subsection{Somatisation}

Somatisation is thought to be related to myofascial pain in the masticatory muscles and to joint-and-muscle related TMD. However, such a connection to joint-related TMD has not been fully established [18]. What is recognized, however, is that the influence of somatisation on chronic painful TMD is not, as it was presumed in the past, associated with an increased parafunctional activity during sleep [35].

\subsection{Compulsive behaviours}

Compulsions are deliberate actions that an individual feels driven to perform; they often accompany recurrent obsessive thoughts. These repetitive and stereotype rituals are neither useful nor pleasant to the patient, and are frequently associated with psychological tension [39]. Some compulsions may also be related to parafunctional activities of the masticatory organ, the most frequent of which is onychophagia.

\subsubsection{Onychophagia}

Habitual nail biting, i.e. onychophagia (from Greek: onycho - nail, phagein - to eat), is classified as a psychodermatosis, the symptoms of which are associated with the expression of certain mental disorders. As a widespread habit, it can be observed both in children and in adults. It is estimated to be practiced within $20-30 \%$ of the population, and in as much as $45 \%$ of young people. Its onset is usually observed at the age of 4-6 years, and its frequency increases with age [14]. According to ICD-10 classification, onychophagia is a specific conduct and emotional disorder that begins in childhood and adolescence, while the Diagnostic and Statistical Manual of Mental Disorders (DMS IV) classifies it as an impulse control disorder [2,39]. This group includes also, for instance, kleptomania and pyromania. There are two types of onychophagia: mild and severe. The aetiology of onychophagia is multifactorial. It is most frequently related to malaise, emotional hypersensivity, low self-esteem, complexes, excitability and apprehensiveness. Onychophagia can be more frequently found in neurotic individuals. Among its causes, the literature also reports: transference from a sucking habit, child's response to unfulfilled basic emotional needs related to the closest person, lack of love and sense of insecurity, as well as abnormal blood levels of iron, copper and vitamins. Prolonged nail biting leads to numerous cosmetic (e.g. michronychia) and health complications, including dental problems: malocclusions, temporomandibular joint disorders, masseter muscle hypertrophy, tooth surface wear and gum inflammations [14]. 


\section{Catastrophizing}

Catastrophizing is one of the basic deformations of thought present in depression and anxiety disorders. Catastrophizing is a set of negative thought practices with regard to future possibilities and met-with situations, with the concurrent expression of strong emotions, especially depression. All of the aforementioned may initiate a focused attention on pain [39].

Catatsrophizing is associated with numerous musculoskeletal disorders, and, as reported by patients, can bring about a marked enhancement in the intensity of pain experiences and related function limitations. Moreover, in individuals with catastrophizing thoughts, pain treatment is usually less effective than in people who do not have such thoughts [7]. This was noted in an experiment that involved injecting 5\% hypertonic salt solution into the masseter muscle: patients with an increased tendency to catastrophizing, report greater intensity and area of pain than did people without such a tendency. It is suggested that this is due to the central sensitization in people with more intense catastrophizing tendencies, which may affect the activity at the higher levels of the central nervous system, including the prefrontal cortex that controls strong emotions [1]. Inclusion of the tools to diagnose catastrophizing in the clinical examination will enable early identification of patients with such a tendency and then enable the choosing of an appropriate treatment approach [17].

\section{CONCLUSION}

The disorders related to stress, anxiety and depression intensify parafunctional activity that may lead to the emergence or exacerbation of TMD. In addition, anxiety-depressive disorders, somatisation and catastrophizing contribute to chronic TMD, mainly in the form of myofascial pain. Knowledge of the influence of psychological factors in TMD enables identification of the patients with an increased risk of chronic painful TMD. Special attention must be given to individuals with depression and catastrophizing tendency, because in this group of patients TMD, and especially its muscle-related form, is observed most frequently.

\section{REFERENCES}

1. Akhter R., Benson J., Svensson P., et al.: Experimental jaw muscle pain increases pain scores and jaw movement variability in higher pain catastrophizers. J. Oral Facial Pain Headache, 28, 205, 2014.

2. American Psychiatric Association (2000). Diagnostic and Statistical Manual of Mental Disorders, Fourth Edition: DSM-IV-TR ${ }^{\oplus}$. American Psychiatric Association; p.996.

3. Brandini D.A., Benson J., Nicholas M.K., et al.: Chewing in temporomandibular disorder patients: An exploratory study of an association with some psychological variables. J. Orofac. Pain, 25, 56, 2011.

4. Carrara S.V., Conti P.C.R., Barbosa J.S.: Statement of the 1st Consensus on Temporomandibular Disorders and Orofacial Pain. Dent. Press J. Orthod., 15, 114, 2010.

5. Costa P.T., McCrae R.R.: Normal personality assessment in clinical practice: The NEO Personality Inventory. Psychol. Assess., 4, 5, 1992.

6. Dworkin S.F., LeResche L.: Research diagnostic criteria for temporomandibular disorders: Review, criteria, examinations and specifications, critique. J. Craniomandib. Disord. Facial Oral Pain, $6,301,1992$.
7. Edwards R.R., Bingham C.O., Bathon J., et al.: Catastrophizing and pain in arthritis, fibromyalgia, and other rheumatic diseases. Arthritis Rheum., 55, 325, 2006.

8. Eitner S., Stingl K., Schlegel A.K., et al.: Biopsychosocial correlations in patients with chronic oro-facial pain. Part II. Experiences of pain and dramatic events before the 16th year of life. J. Oral Rehabil., 36, 408, 2009.

9. Fernandes G., Gonçalves D.A. de G., de Siqueira J.T.T. de, et al.: Painful temporomandibular disorders, self reported tinnitus, and depression are highly associated. Arq. Neuropsiquiatr., 71, 943, 2013.

10. Fillingim R.B., Ohrbach R., Greenspan J.D., et al.: Psychological factors associated with development of TMD: the OPPERA prospective cohort study. J. Pain, 14, 12 Suppl, T 75-90, 2013.

11. Fillingim R.B., Ohrbach R., Greenspan J.D., et al.: Potential psychosocial risk factors for chronic TMD: descriptive data and empirically identified domains from the OPPERA case-control study. J. Pain, 12, 11 Suppl, T 46-60, 2011.

12. Fischer W.F., O'toole E.T.: Personality characteristics of chronic bruxers. Behav. Med., 19, 82, 1993.

13. Funato M., Ono Y., Baba K., et al.: Evaluation of the non-functional tooth contact in patients with temporomandibular disorders by using newly developed electronic system. J. Oral Rehabil., 41, 170, 2014.

14. Gniadek A., Czochara J.: Konsekwencje zdrowotne onychofagii. Hygeia Public Health., 45, 109, 2010.

15. Goesling J., Clauw D.J., Hassett A.L.: Pain and depression: An integrative review of neurobiological and psychological factors. Curr. Psychiatry Rep., 15, 421, 2013.

16. Guarda-Nardini L., Pavan C., Arveda N., et al.: Psychometric features of temporomandibular disorders patients in relation to pain diffusion, location, intensity and duration. J. Oral Rehabil., 39, 737, 2012.

17. Haythornthwaite J.A.: IMMPACT recommendations for clinical trials: Opportunities for the RDC/TMD. J. Oral Rehabil., 37, 799, 2010.

18. Huang G.J., LeResche L., Critchlow C.W., et al.: Risk Factors for Diagnostic Subgroups of Painful Temporomandibular Disorders (TMD). J. Dent. Res., 81, 284, 2002.

19. Kindler S., Samietz S., Houshmand M., et al.: Depressive and anxiety symptoms as risk factors for temporomandibular joint pain: A prospective cohort study in the general population. J. Pain, 13, $1188,2012$.

20. Lajnert V., Francisković T., Grzic R., et al.: Depression, somatization and anxiety in female patients with temporomandibular disorders (TMD). Coll. Antropol., 34, 1415, 2010.

21. Leary M.R., Hoyle R.H. (2013). Handbook of Individual Differences in Social Behavior. Guilford Publications; p.642.

22. Lobbezoo F., Ahlberg J., Glaros A.G., et al.: Bruxism defined and graded: an international consensus. J. Oral Rehabil., 40, 2, 2013.

23. Main C.J., Williams A.C. de C.: Musculoskeletal pain. BMJ., 325, $534,2002$.

24. Maísa Soares G., Rizzatti-Barbosa C.M.: Chronicity factors of temporomandibular disorders: A critical review of the literature. Braz. Oral Res., 29, 1, 2015.

25. Manfredini D., Cantini E., Romagnoli M., et al.: Prevalence of bruxism in patients with different research diagnostic criteria for temporomandibular disorders (RDC/TMD) diagnoses. Cranio J. Craniomandib. Pract., 21, 279, 2003.

26. Manfredini D., Lobbezoo F.: Role of psychosocial factors in the etiology of bruxism. J. Orofac. Pain., 23, 153, 2009.

27. Matud M.P.: Gender differences in stress and coping styles. Personal Individ. Differ., 37, 1401, 2004.

28. McCrae R.R., Costa P.T.: Personality trait structure as a human universal. Am. Psychol., 52, 509, 1997.

29. Merskey H., Bogduk N. (1994). Classification of Chronic Pain: Descriptions of Chronic Pain Syndromes and Definitions of Pain Terms. IASP Press; p.244.

30. Moayedi M., Weissman-Fogel I., Crawley A.P., et al.: Contribution of chronic pain and neuroticism to abnormal forebrain gray matter in patients with temporomandibular disorder. NeuroImage, 55, 277, 2011. 
31. Pallegama R.W., Ranasinghe A.W., Weerasinghe V.S., et al.: Anxiety and personality traits in patients with muscle related temporomandibular disorders. J. Oral Rehabil., 32, 701, 2005.

32. Pingitore G., Chrobak V., Petrie J.: The social and psychologic factors of bruxism. J. Prosthet. Dent., 65, 443, 1991.

33. Reissmann D.R., John M.T., Schierz O., et al.: Stress-related adaptive versus maladaptive coping and temporomandibular disorder pain. J. Orofac. Pain., 26, 181, 2012.

34. Reissmann D.R., John M.T., Seedorf H., et al.: Temporomandibular disorder pain is related to the general disposition to be anxious. $J$. Oral Facial Pain Headache, 28, 322, 2014.

35. Shedden Mora M., Weber D., Borkowski S., et al.: Nocturnal masseter muscle activity is related to symptoms and somatization in temporomandibular disorders. J. Psychosom. Res., 73, 301, 2012.
36. Shephard M.K., Macgregor E.A., Zakrzewska J.M.: Orofacial pain: a guide for the headache physician. Headache, 54, 22, 2014.

37. Szabo S., Tache Y., Somogyi A.: The legacy of Hans Selye and the origins of stress research: A retrospective 75 years after his landmark brief "letter" to the editor of nature. Stress Amst. Neth., 15, 472, 2012.

38. Wieckiewicz M., Grychowska N., Wojciechowski K., et al.: Prevalence and correlation between TMD based on RDC/TMD diagnoses, oral parafunctions and psychoemotional stress in Polish university students. Biomed Res. Int., 2014, ID 472346, 2014.

39. World Health Organization. (2004). International Statistical Classification of Diseases and Related Health Problems. World Health Organization; p. 824. 